\title{
Coupling strength of charge carriers to spin fluctuations in high-temperature superconductors
}

\author{
J.P. Carbotte ${ }^{\star}$, E. Schachinger ${ }^{\dagger}$ \& D.N. Basov ${ }^{\ddagger}$ \\ ${ }^{\star}$ Department of Physics and Astronomy, McMaster University \\ Hamilton, Ont. L8S 4M1, Canada \\ ${ }^{\dagger}$ Institut für Theoretische Physik, Technische Universität Graz \\ A-8010 Graz, Austria \\ ${ }^{\ddagger}$ Physics Department, University of California - San Diego \\ La Jolla California 92093-0319, U.S.A.
}

(April 24, 2022)

In conventional superconductors, the most direct evidence of the mechanism responsible for superconductivity comes from tunnelling experiments in which a clear image of the electronphonon interaction is revealed. structure in the current voltage characteristics at the phonon energies can be used to measure, through inversion of the Eliashberg equations, the electron phonon spectral density $\alpha^{2} F(\omega)$. 1 The coherence length in conventional materials is long and the tunnelling process probes several atomic layers into the bulk of the superconductor. On the contrary, in the high $T_{c}$ oxides, particularly for $c$-axis tunneling, the coherence length can be quite short and in an optical experiment or in neutron scattering experiments the bulk of the sample is probed. Therefore, these spectroscopies become the methods of choice for the investigation of mechanisms of high- $T_{c}$ superconductivity. Accurate reflectance measurements in the infrared range and precise polarized neutron scat tering data are available for a variety of oxides. 3 In this paper we show that conducting carriers studied by means of infrared spectroscopy reveal strong coupling to a resonance structure in the spectrum of spin fluctuations examined with neutron scattering. The coupling strength inferred from experiment is sufficient to account for high values of $T_{c}$ which signals the prominent role of spin excitations in the superconductivity of oxides.

There have been many suggestions as to the mechanism involved in the superconductivity of the oxides. While, so far, no consensus has yet emerged, the state itself is widely accepted to have $d$-wave symmetry 9 with the gap on the Fermi surface vanishing along the main diagonals of the two-dimensional Brillouin zone. In YBCO there also exists extensive spin polarized inelastic neutron scattering data. These experiments reveal that spin excitations persist on a large energy scale 10 over several $100 \mathrm{meV}$, but are mainly confined around the $(\pi, \pi)$ point in momentum space. Also, in the superconducting state, a new peak emerges out of, or is additional to, the spin excitation background which is often referred to as the $41 \mathrm{meV}$ resonance司 (Fig. 1). This peak has regeired much attention but its origin remains uncertain. $11.12 \mathrm{In}$ one view, it is due to a readjustment in the spin exfitation spectrum due to the onset of superconductivity.11 Such a readjustment of spectral weight with a reduction below twice the superconducting gap value $\Delta_{0}{ }^{13} \mathrm{H}_{14}$ is expected on general ground and is generic to electronic mechanisms. A second view is that it is a resonance in the $S O(5)$ unification 12 of magnetism and superconductivity.

If the spin excitations are strongly coupled to the charge carriers they should be seen in optical experiments. The normal state optical conductivity $\sigma(\omega)$ as a function of frequency $(\omega)$ depends on the electron self energy $\Sigma(\omega)$ which describes the effect of interactions on electron motion. In an electron-phonon system the electron-phonon interaction spectral density, $\alpha^{2} F(\omega)$, is

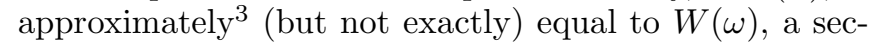
ond derivative of the inverse of the normal state optical conductivity

$$
\alpha^{2} F(\omega) \approx W(\omega)=\frac{1}{2 \pi} \frac{d^{2}}{d \omega^{2}}\left[\omega \Re \mathrm{e} \frac{1}{\sigma(\omega)}\right]
$$

In the phonon energy range, the correspondence is remarkably close and determines $\alpha^{2} F(\omega)$ with good accuracy. At higher energies, additional, largely negative wiggles come into $W(\omega)$ which can simply be ignored as they are not part of $\alpha^{2} F(\omega)$. Note that (1) is dimensionless and so determines the absolute scale of the electron-phonon interaction spectral density as well as its shape in frequency. This fact is important as it allowed Marsiglio et al. 3 to determine the $\alpha^{2} F(\omega)$ of $\mathrm{K}_{3} \mathrm{C}_{60}$ from its optical conductivity by inversion (11) and to conclude from a solution of the Eliashberg equations that it is large enough to explain the observed value of critical temperature. $\left(T_{c}\right.$ is related to the mass enhancement factor $\lambda$, twice the first inverse moment of $\alpha^{2} F(\omega)$.2)

The formalism for the normal state conductivity can also be applied to spin excitations. If we ignore anisotropy as a first approximation, we can proceed by introducing an electron-spin excitation spectral density denoted by $I^{2} \chi(\omega)$ with its scale set by the coupling strength to the charge carriers, $I^{2}$, and $\chi(\omega)$ the imaginary part of the spin susceptibility measured in spin 


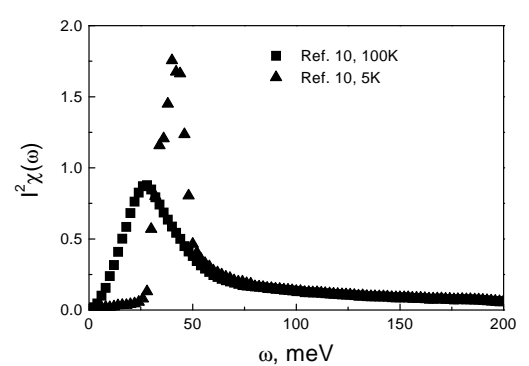

FIG. 1. Spin polarized inelastic neutron scattering data for $\mathrm{YBa}_{2} \mathrm{Cu}_{3} \mathrm{O}_{6.92}$. Neutron scattering results taken at $100 \mathrm{~K}$ in the normal state (solid squares) compared with similar data at $5 \mathrm{~K}$ in the superconducting state (solid triangles) showing the $41 \mathrm{meV}$ resonance. The data have been scaled by a constant coupling strength $I^{2}$ fixed to get a $T_{c}=100 \mathrm{~K}$.

polarized inelastic neutron scattering experiments averaged over all momentum in the Brillouin zone. At low temperatures $\chi(\omega)$ contains the $41 \mathrm{meV}$ resonance observed in the superconducting state. To illustrate our main point we will use here $\chi(\omega)$ directly from experimental results on a $\mathrm{YBa}_{2} \mathrm{Cu}_{3} \mathrm{O}_{6.92}$ sample with $T_{c}=$ $91 \mathrm{~K}$, near optimum doping and for which results exist at the temperatures $T=100 \mathrm{~K}$ and $T=5 \mathrm{~K}, 10$ both properly calibrated in units of $\mu_{B}^{2} / \mathrm{eV}\left(\mu_{B}\right.$ is the Born magneton) as shown in Fig. 且. We multiply $\chi(\omega)$ at $T=100 \mathrm{~K}$ by a constant coupling $I^{2}$ fixed to get $T_{c}=100 \mathrm{~K} .5$ The mass enhancement factor $\lambda$ (twice the first inverse moment of $\left.I^{2} \chi(\omega)\right)$ obtained is 2.6 and is, to within 10 percent, the same as obtained from the $W(\omega)$ derived from the normal state experimental data of Basov et al 19 in $\mathrm{YBa}_{2} \mathrm{Cu}_{3} \mathrm{O}_{6.95}$ and from our calculated $W(\omega)$ at $T_{c}$. In a preliminary attempt to invert Collins et al. 17 found a $\lambda$ of three which is greater than our value. Their twinned crystals exhibited a higher optical scattering rate than our untwinned crystal and consequently they obtained about $50 \%$ more weight in the main peak of $I^{2} \chi(\omega)$ around $30 \mathrm{meV}$.

In order to access lower temperatures, we need to understand how the $I^{2} \chi(\omega)$ structure enters the superconducting state optical conductivity. To this end, we have done a series of calculations of the superconducting state $\sigma(\omega)$ for a $d$-wave superconductor including inelastic scattering. Detaily have been presented in the work of Schachinger et al. 15 We used their prescription to calculate the theoretical $\sigma(\omega)$ using the neutron data taken for $\mathrm{YB}_{2} \mathrm{C}_{3} \mathrm{O}_{6.92}$ (at $5 \mathrm{~K}$ ) as $\chi(\omega)$ multiplied by the same value of the coupling strength $I^{2}$ previously determined to obtain a $T_{c}$ of $100 \mathrm{~K}$ from the normal state neutron data. We then inverted this theoretical $\sigma(\omega)$ data using Eq. (1). The result of this inversion is compared in the top frame of Fig. 2 (solid line) with our input spectral density $I^{2} \chi(\omega)$ (solid triangles) shifted in energy-by the gap $\Delta_{0}=27 \mathrm{meV}$ of our theoretical calculations.18

The absolute scale of $I^{2} \chi(\omega)$ in the resonance region is well given by the peak value in the solid curve. This peak is followed by negative wiggles which are not in the
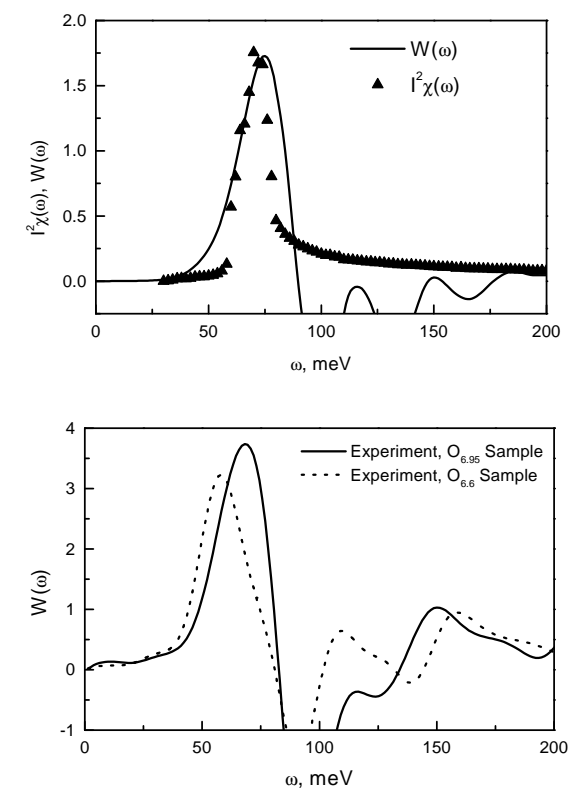

FIG. 2. Inversion of the superconducting state optical conductivity. Results (top frame) for $W(\omega)$ (solid curve) with $\sigma(\omega)$ calculated in the superconducting state at $5 \mathrm{~K}$ and based on the spectral density $I^{2} \chi(\omega)$ (solid triangles) equal to the $5 \mathrm{~K}$ measured neutron data. In the region of the main resonance peak, the agreement with the input data, which has been shifted by the gap value (solid triangles), is excellent as to height and width. At higher energies, wiggles appear in $W(\omega)$ which are not present in $I^{2} \chi(\omega)$. Bottom frame, $W(\omega)$ derived from the experimental data for the conductivity of an optimally doped $\mathrm{YBa}_{2} \mathrm{Cu}_{3} \mathrm{O}_{6.95}$ single crystal with $a$-polarization (solid curve) and for an underdoped, untwinned $\mathrm{YBa}_{2} \mathrm{Cu}_{3} \mathrm{O}_{6.6}$ single crystal (dashed line).

original input spectrum because $W\left(\omega+\Delta_{0}\right)$ is not exactly $I^{2} \chi(\omega)$. Nevertheless, such a procedure allows us to see quite directly by spectroscopic means some of the features of $I^{2} \chi(\omega)$ and, more importantly gives us information on its absolute value at maximum. The long tails in $I^{2} \chi(\omega)$ at higher energy extending well beyond the resonance are not resolved in $W(\omega)$ but cause $\tau^{-1}(\omega)$, defined as $\Re \mathrm{e}\left\{\sigma^{-1}(\omega)\right\}$, to rise in a quasi linear fashion at high frequenciest in both normal and superconducting state, as is observed. This quasilinear rise mas the motivation for the marginal Fermi liquid mode 14 which gives $\tau^{-1}(\omega) \propto \omega$ and a constant spectral density for $\omega>T$ extending to high energies. If we approximate the normal state experimental $\tau^{-1}(\omega)$ datal at $T_{c}$ by a straight line for $0 \leq \omega \leq 200 \mathrm{meV}$, we get 0.3 as the weight of the spectral density for all frequencies $\omega>T$ and a $\lambda$ of 2.8 quite consistent with our previous estimates. It is important to realize that, in as much as the $41 \mathrm{meV}$ resonance is near $2 \Delta_{0}$, the density of quasiparticle states (not shown here), has structure at $3 \Delta_{0}$ in our calculations, a well established feature of tunnelling data particularly in Bi2212.19

In the bottom frame of Fig. 2 we show experimental 
results obtained from the data by Basov et al. 16 on application of the prescription (11) to $a$-axis conductivity data on an untwinned single crystal. The $41 \mathrm{meV}$ resonance is clearly resolved as a peak at approximately 69 $\mathrm{meV}$ in the solid curve (the gap is $27 \mathrm{meV}$ ). The height of this peak is about 3 and gives an absolute measure of the coupling between charge carriers and spin excitations. On comparison with the top frame of Fig. 2, we see that the coupling to the $41 \mathrm{meV}$ resonance is larger in the experiment than the value assumed in the calculations that generated the theoretical results of that frame. This is not surprising since we have used the spin polarized inelastic neutron scattering data set measured on a near optimum $91 \mathrm{~K}$ sample of $\mathrm{YBa}_{2} \mathrm{Cu}_{3} \mathrm{O}_{6.92}$ while the neutron results for slightly overdoped YBCO are very different 5 although the $T_{c}$ value is hardly affected. This large dependence of $\chi(\omega)$ on the sample can be used to argue against their role in establishing $T_{c}$. However, the function that controls the conductivity is a complicated weighting of the spin susceptibility involving details of the Fermi surface and points in the Brillouin zone away from $(\pi, \pi)$ as well as the coupling to the charge carriers. Thus, the correspondence between $I^{2} \chi(\omega)$ and $\chi(\omega)$ is complicated. What optical experiments reveal is that $I^{2} \chi(\omega)$ is not as strongly dependent on doping as is $\chi(\omega)$.

In Fig. 2, bottom frame, we present experimental results for $W(\omega)$ in underdoped, untwinned $\mathrm{YB}_{2} \mathrm{C}_{3} \mathrm{O}_{6.6}$ (dashed line) and compare with the optimally doped case (solid line). It is interesting to note that the peak in the underdoped case is slightly reduced in height reflecting a reduction in $T_{c}$. It is also shifted to lower energies. Some experiment 20 indicate a reduction in gap value with underdoping in YBCO while many experiments show an important increase in Bi2212.19 Even if the gap is assumed to stay the same at $27 \mathrm{meV}$, the spin polarized neutron resonant frequency is known to decrease with doping. 21 Accounting for this gives almost exactly the downward shift observed in our experimental data of Fig. 2 (bottom frame).

Very recently, inelastic neutron scattering data in Bi221222 have been published. They show a resonance peak at $43 \mathrm{meV}$ in the superconducting state and establish a similarity with the earlier results in YBCO. We have inverted the optical data of Puchkov et al. 1 in this case and find that coupling at low temperatures to the observed superconducting state spin resonance peak is a general phenomena in both YBCO and Bi2212.

Spin excitations are seen in an appropriately chosen second derivative of the superconducting state optical conductivity and the strength of their coupling to the charge carriers determined from such data. The coupling to the excitations including the $41 \mathrm{meV}$ resonance is large enough in YBCO that it can account for superconductivity at that temperature. At $T_{c}$ the spectrum obtained from experiment $t$ gives a value of the mass enhancement parameter $\lambda$ which is close to the value used in our model calculations to obtain a critical temperature of $100 \mathrm{~K}$.
${ }^{1}$ McMillan, W.L. and Rowell, J.M. Lead Phonon Spectrum Calculated from Superconducting Density of States, Phys. Rev. Lett. 14, 108-112 (1965).

${ }^{2}$ Carbotte, J.P. Properties of Boson Exchange Superconductors, Rev. Mod. Phys. 62, 1027-1157 (1990).

${ }^{3}$ Marsiglio, F., et al. Inversion of $\mathrm{K}_{3} \mathrm{C}_{60}$ Reflectance Data, Phys. Lett. A245, 172-176 (1998).

${ }^{4}$ Puchkov, A.V., et al. High $T_{c}$ Superconductors: an infrared study, J. Phys. Condens. Matter 8, 10049-10082 (1996).

${ }^{5}$ Bourges, P. From Magnons to the Resonance Peak: Spin Dynamics in High- $T_{c}$ Superconducting Cuprates by Inelastic Neutron Scattering, in: The Gap Symmetry and Fluctuations in High Temperature Superconductors, ed: J. Bok, G. Deutscher, D. Parrina, and S.A. Wolf, Plenum Press 1998, preprint cond-mat/9901333.

${ }^{6}$ Scalapino, D.J., et al. $d$-Wave Pairing Near a Spin-DensityWave Instability, Phys. Rev. B 34, 8190-8192 (1986).

${ }^{7}$ Monthoux, P. and Pines, D. YBCO: A Nearly Antiferromagnetic Fermi Liquid, Phys. Rev. B 47, 6069-6081 (1993).

${ }^{8}$ Wollmann, D.A. et al. Experimental Determination of the Superconducting Pairing State in YBCO from the Phase Coherence of YBCO-Pb dc SQUIDs, Phys. Rev. Lett. 71, 2134-2137 (1993).

${ }^{9}$ Tsuei, C.C. et al. Pairing Symmetry and Flux Quantization in a Tricrystal Superconducting Ring of YBCO, Phys. Rev. Lett. 73, 593-596 (1994).

${ }^{10}$ Bourges, P. el al. Spin dynamics in high- $T_{c}$ superconductors, preprint cond-mat/9902067.

${ }^{11}$ Bulut, N. and Scalapino, D.J. Neutron Scattering from a Collective Spin Fluctuation Mode in a $\mathrm{CuO}_{2}$ Bilayer, Phys. Rev. B 53, 5149-5152 (1996).

${ }^{12}$ Demler, E. and Zhang, S.C. Theory of the Resonant Neutron Scattering of High- $T_{c}$ Superconductors, Phys. Rev. Lett. 75, 4126-4129 (1995).

${ }^{13}$ Nuss, M.C. et al. Dynamic Conductivity and "Coherence Peak" in $\mathrm{YBa}_{2} \mathrm{Cu}_{3} \mathrm{O}_{7}$ Superconductors, Phys. Rev. Lett. 66, 3305-3308 (1991).

${ }^{14}$ Varma, C.M. et al. Phenomenology of the Normal State of Cu-O High Temperature Superconductors, Phys. Rev. Lett. 63, 1996-1999 (1989).

${ }^{15}$ Schachinger, E. et al. Suppression of Inelastic Scattering on Penetration Depth and Conductivity in $d_{x^{2}-y^{2}}$ Superconductors, Phys. Rev. B 56, 2738-2750 (1997).

${ }^{16}$ Basov, D.N. et al. Pseudogap and Charge Dynamics in $\mathrm{CuO}_{2}$ Planes in YBCO, Phys. Rev. Lett. 77, 4090-4093 (1996).

17 Collins, R.T. et al. Reflectivity and conductivity of $\mathrm{YBa}_{2} \mathrm{Cu}_{3} \mathrm{O}_{7}$, Phys. Rev. B 39, 6571-6574 (1989).

${ }^{18}$ Marsiglio, F. et al. to be published.

${ }^{19}$ DeWilde, Y. et al. Unusual Strong Coupling Effects in the Tunneling Spectroscopy of Optimally Doped and Overdoped $\mathrm{Bi}_{2} \mathrm{Sn}_{2} \mathrm{CaCu}_{2} \mathrm{O}_{8+\delta}$, Phys. Rev. Lett. 80, 153-156 (1998).

${ }^{20}$ Ponomarev, Yo.G. et al. Josephson Effect and Single Par- 
ticle Tunneling in YBCO and YBCO Single Crystal Break Junctions, Physica C 243, 167-176 (1995).

${ }^{21}$ Fong, H.F. et al. Superconducting-Induced Anomalies in the Spin Excitation Spectra of Underdoped YBCO, Phys. Rev. Lett. 78, 713-716 (1997).

${ }^{22}$ Fong, H.F. et al. Neutron Scattering from Magnetic Excitations in $\mathrm{Bi}_{2} \mathrm{Sr}_{2} \mathrm{CaCu}_{2} \mathrm{O}_{8+\delta}$, Nature 398, 588 (1999).

Acknowledgments. This work was supported in part by the Natural Sciences and Engineering Research Council of Canada (NSERC), the Canadian Institute for Advanced Research (CIAR). Work at UCSD is supported by the NFS "Early Career Development" program. DNB is a Cottrel Scholar of the Research Corporation. We thank J.E. Hirsch, P.B. Hirschfeld, P.B. Littlewood, F. Marsiglio, E.J. Nicol, D. Scalapino, T. Timusk, and I. Vekhter for interest. 